\section{Révision partielle de la loi fédérale sur l'assurance-maladie}

\author{
Projet de révision du 18 septembre 2000
}

H. H. Brunner, président de la FMH

Le message du 18 septembre 2000 relatif à la révision partielle de la loi fédérale sur l'assurance-maladie (ci-après "message») est actuellement examiné par la Commission de la sécurité sociale et de la santé publique (CSSS) du Conseil des Etats, première chambre dans ce dossier. A cet égard, dissipons un malentendu largement répandu dans le corps médical: en tant que projet, le message n'est soumis à aucune consultation, celle-ci ayant déjà eu lieu en préliminaire à ce message [1]. Dans le cadre de ces travaux préparatoires cependant, une audition a été tenue par la CSSS, à laquelle ont aussi participé certains conseillers nationaux. La FMH a eu l'occasion de présenter la position résumée ci-dessous et, sur la base des discussions qui ont suivi, d'analyser l'état des lieux en matière de débat politique, dans la perspective, surtout, de ses possibles développements. Les lignes qui suivent résument cette position et cette analyse. Pour une meilleure compréhension, nous renvoyons au texte $d u$ projet de révision publié en fin d'article.

Hormis les adaptations qui sont devenues indispensables - principalement en raison de la jurisprudence - ou celles ne présentant qu'une importance mineure, le projet de révision contient d'une part des propositions visant à modifier les modalités de financement dans la LAMal dont les effets s'étendraient bien audelà du domaine hospitalier. D'autre part, il envisage des modalités de limitation de l'obligation de contracter.

Le Conseil fédéral a, hélas, refusé le pas constamment demandé par la FMH, entre autres, vers un financement hospitalier de type moniste. Le système dualiste demeure (financement par l'assureur et les pouvoirs publics à $50 \%$ fixes), un système qui empêche une limitation claire des responsabilités et, par là-même, une gestion efficace (des coûts). Les pouvoirs publics, les cantons en particulier, conservent leur triple rôle de propriétaires, de financiers et d'autorités de surveillance des hôpitaux. Nombre de volteface incompréhensibles pour l'usager normal, dans le domaine de la santé publique, trouvent ici leur explication.

Saluons la démarche tentée, du moins, pour passer du financement de l'objet à celui de la prestation, en conformité, disons-le, avec la toujours actuelle revendication de la FMH d'un traitement égalitaire de l'hôpital et du cabinet médical. Plusieurs problèmes peuvent ainsi trouver leur solution:
- Les termes qui attisent la polémique, tels que «division commune" ou "division privée», deviennent caducs. L'essentiel, concernant les principes et les modalités selon la LAMal, est de savoir si les prestations relèvent du catalogue des prestations de la LAMal, ce qui rappelle une autre exigence de la FMH, à savoir celle d'une meilleure définition terminologique.

Rien ne sert de vouloir exorciser le mal par le mal en créant de nouvelles imprécisions. Je veux parler de la définition du terme "traitement semihospitalier", que les plus éminents spécialistes de politique de la santé de ce pays n'auront pas réussi à donner en quinze années d'efforts (sauf pour le domaine psychiatrique). Logiquement, des prestations ne peuvent être réparties qu'en deux catégories, selon qu'elles sont ambulatoires (le patient dort chez lui) ou hospitalières (le patient dort tant que faire se peut à l'hôpital). Il n'y a pas de troisième possibilité. Donner aux hôpitaux, par des termes tels que semi-hospitalier, le prétexte pour vendre plus cher une prestation, est un mensonge à courte vue que les chiffres ne tardent pas à révéler au grand jour.

- Le principe jusqu'ici valable de la couverture des coûts ou du remboursement des coûts a conduit à ce que les tarifs hospitaliers n'indemnisent qu'une partie des coûts facturables, qui plus est d'après des modalités différentes selon les cantons, entraînant par là-même des prix différents de canton à canton pour une même prestation, sans compter les divergences d'avec les tarifs ou les prix en pratique privée. L'introduction du TarMed devrait changer cet état de choses. Il convient dès lors de saluer la résolution de ce problème grâce à la rémunération de la prestation selon un système tarifaire unifié pour l'ensemble de la Suisse, dans le domaine hospitalier également, avec des forfaits par cas qui devraient peu après faire leur apparition dans l'ensemble du secteur ambulatoire.

\section{Intervention du DFI dans l'assurance complémentaire}

Depuis l'introduction de la nouvelle LAMal, on entend la critique, de la part de milieux médicaux également, selon laquelle l'assuré complémentaire a droit également, pour un traitement hospitalier, à une indemnisation au prorata de la part de l'assurance de base, à laquelle il est tenu de s'affilier selon la loi. Le TFA a soutenu cette thèse, à vrai dire dans le cas d'un traitement hors canton. Même en l'absence de jugements de haute instance relatifs au traitement dans le canton de domicile du patient, l'application de ce principe pour le traitement de patients au bénéfice de l'assurance complémentaire dans le canton de domicile est logiquement obligatoire. Le message ancre désormais ce principe dans la loi (cf. art. 49, en particulier les $1^{\text {er }}$ et $2^{\mathrm{e}}$ al.). 
Pour le médecin qui traite des patients au bénéfice de l'assurance complémentaire, il est dès lors essentiel de connaître le $5^{\mathrm{e}}$ al. de cet article 49 qui stipule que toutes les prétentions de l'hôpital (et, dans la foulée, celles du médecin traitant) sont rémunérées selon les alinéas 1 à 4 de l'article 49. En clair et en résumé: la part de l'assurance de base aux traitements hospitaliers fait que l'ensemble des prestations de la LAMal est importé dans l'assurance complémentaire d'une manière qui exclut toute facturation de ces prestations via l'assurance complémentaire. Ce qui demeure du domaine d'indemnisation de l'assurance complémentaire selon les structures tarifaires actuelles est constitué par l'hôtellerie et les prestations non prises en charge telles que le remodelage facial, les opérations purement esthétiques des seins, les transplantations capillaires, etc.

Il va sans dire que le département de Mme Dreifuss a posé ici un jalon dans sa croisade contre l'assurance complémentaire, une croisade habilement orchestrée et médiatisée, planifiée et menée systématiquement depuis des années, sans trêve jusqu'à ce jour. Dans ce contexte, il devrait être extrêmement difficile de freiner cette dramatique évolution, c'està-dire de radier les dispositions législatives en question, d'autant que certains groupements au sein du corps médical lui ont largement ouvert la voie avec leurs revendications visant à lier l'assurance complémentaire à l'assurance de base, tant sur le plan tarifaire que légal. Depuis près de dix ans, le Comité central de la FMH n'a cessé ses mises en garde contre cette prévisible évolution.

Qu'en est-il aujourd'hui? La réponse à cette question est simple, au fond: l'assurance complémentaire doit rembourser les prestations qui ne relèvent pas du domaine de la LAMal et de sa protection tarifaire. Concrètement, cela revient à dire que:

- la règle de base ne saurait demeurer la rémunération plus élevée des prestations couvertes par les tarifs de l'assurance sociale (TarMed!);

- l'assurance complémentaire rembourse la prestation médicale personnelle du médecin, choisi par le patient assuré complémentaire selon le principe du libre choix du médecin pour le traitement en question;

- l'éventail des prestations de l'assurance sociale de base doit être réduit dans le but de permettre à l'assurance sociale de base de financer principalement les traitements coûteux (une idée guère nouvelle, au demeurant, mais qui devrait enfin trouver son application).

Des modèles d'assurance complémentaire de ce genre ont déjà été conçus; ils ont retenu l'attention des assureurs complémentaires, dont les réflexions vont dans le même sens. Un élément décisif est que les travaux ne peuvent plus constituer la prérogative soigneusement préservée de certains groupements de médecins poursuivant des objectifs secondaires, mais doivent se fonder sur un consensus de l'ensemble du corps médical.

\section{Limitation de l'obligation de conclure une convention}

La limitation de l'obligation de contracter proposée en consultation n'a trouvé d'accord - rien d'étonnant à cela - qu'auprès des assureurs maladie. Selon le message, cette limitation de l'obligation de contracter devrait désormais s'appliquer uniquement aux fournisseurs de prestations qui ont dépassé l'âge de 65 ans. Le motif esquissé dans le texte d'accompagnement, selon lequel cette réglementation contribuerait à l'assurance-qualité, est si peu convaincant et d'une telle mesquinerie qu'il ne parvient pas à cacher les véritables intentions. Celles-ci sont d'ancrer aux dépens des anciens, dont on n'attend guère de réactions et qui ne disposent d'aucun groupe de pression, le principe de la levée de l'obligation de contracter selon le diktat des assureurs-maladie de manière à pouvoir l'étendre d'ici peu - égalité de traitement oblige - à tout le corps médical, donc également aux jeunes médecins à qui l'on faisait espérer de meilleures perspectives d'avenir si les anciens confrères étaient exclus du système. Un espoir disproportionné qui devrait à très court terme s'évanouir...

Relevons les points suivants en particulier:

- La réglementation toucherait entre 800 et 900 médecins qui accomplissent encore un pensum d'environ 30\% en moyenne (normes TarMed). Ces médecins ne fournissent pratiquement que des prestations attribuées, sur le plan tarifaire, aux dites prestations de base générales. Les activités opératoires et interventionnelles ne représentent que l'exception qui, pour bienvenue qu'elle soit, n'en demeure pas moins rare.

- Il n'existe pas de données qui documenteraient, chez les fournisseurs de prestations selon la LAMal, la perte des facultés intellectuelles et manuelles à partir de l'âge de 65 ans. La conviction selon laquelle de telles limites n'existent pas ont présidé à la création, un peu partout, de "retraites à la carte" dont seraient précisément exclus les "fournisseurs de prestations médicales». Détail piquant: s'agissant de la médecine d'urgence qui n'est pas couverte par l'obligation de contracter, les médecins tombés dans la démence sénile en vertu de ce message semblent retrouver illico toutes leurs facultés ...

- Rien ne force les patients à consulter des médecins plus âgés. L'élimination de la "vieille garde», constituerait aux yeux des patients concernés une destruction des bonnes relations avec leur médecin, sans compter qu'une telle situation serait en complète contradiction avec les règles tant prônées par le législateur de la libre concurrence.

- Le corps médical a toujours conservé le sentiment d'appartenir aux professions libérales, une notion qui présuppose, notamment, l'autodétermination. L'un des points essentiels de cette profession est que ses membres déterminent eux-mêmes la teneur et la durée de leur activité professionnelle. 
Le projet fixerait de facto ces modalités dans la loi, ce qui aurait pour effet de fonctionnariser encore davantage la profession médicale. Nombreux sont ceux qui, approuvant cette fonctionnarisation pour des motifs pas toujours aussi nobles qu'il n'y paraît, ne se posent guère la question de savoir s'ils seraient contents d'avoir à leur propre chevet des médecins issus d'une telle évolution.

Dans le corps médical en général et au sein de la FMH en particulier, on a toujours exigé que les critères pour l'exclusion de médecins des contrats d'assurance soient clairement définis. De tels critères et leur mise en place systématique sont dès lors fortement souhaités par la FMH, ne serait-ce que pour éviter les limitations abusives de l'obligation de contracter. Les critères d'exclusion formulés à l'article 59 du message soulèvent cependant plus de questions qu'ils n'en résolvent.

- Que doit-on comprendre sous le terme de polypragmasie? Qui en donne une définition détaillée? Quelles sont en particulier les données fondant de telles décisions? Après les douloureuses expériences faites avec la procédure de remboursement, la FMH ne saurait tolérer ici de formules nébuleuses.

- Le non-respect de la protection tarifaire sera un motif d'exclusion problématique aussi longtemps que cette protection tarifaire demeurera dans le flou et que nombre de décisions de justice seront encore pendantes.

- Le devoir d'information place le médecin entre Charybde et Scylla, à savoir entre la protection des données et l'exigence de l'assureur alors que la loi ne lui indique pas, comme à Ulysse, le stratagème lui permettant de sortir de l'impasse.

- Venons-en à la non-répercussion d'avantages. Une fois de plus, le législateur évite de donner forme et couleur à cette expression fumeuse telle un vaisseau fantôme, avec pour conséquence de rendre impossible son application. Une fois de plus, on peut s'en servir comme d'un reproche médiatiquement transposable au corps médical. Une fois de plus, tel est là peut-être aussi le but de toute l'opération.

Sous cette forme, la FMH rejette ces dispositions, qu'elle considère comme forcément inefficaces et plaçant le corps médical dans la position kafkaïenne de l'éternel accusé dans l'impossibilité de se défendre.
Le sort de ce projet est actuellement en suspens. Il rencontre un rejet massif des cantons qui craignent, non sans raison, une diminution de leurs prérogatives et des charges supplémentaires entraînées par l'exécution de ses dispositions. L'opposition de nombre de parlementaires, surtout des partis bourgeois, n'est pas moins virulente, ce qui fait plâner sur le projet la menace d'un rejet complet ou - de facto - partiel.

Si le rejet du projet aurait pour effet d'éliminer d'incommodes dispositions pour le corps médical, il ne s'agirait au mieux que d'un gain de temps discutable et il n'y aurait aucune raison de s'en réjouir outre mesure. La grande majorité des parlementaires sous la coupole éprouvent un malaise de plus en grand devant la situation actuelle de la santé publique en Suisse, un malaise qui nécessite à leurs yeux une thérapie de la part des instances confédérales. Pour le corps médical, cette thérapie risque de faire mal.

Le projet se targue de contribuer de manière décisive à un modèle de santé publique concurrentiel. Encore faudrait-il savoir si ce modèle concurrentiel constitue somme toute un apport utile à la gestion des coûts. Difficile de répondre positivement à cette question, aucun pays n'ayant pu jusqu'ici obtenir des preuves à l'appui d'une telle hypothèse. La question va plus loin encore: est-il en définitive admissible, selon des considérations d'ordre politique et éthique, de structurer la santé publique selon un modèle fondé sur la concurrence? Les prestations médicales sontelles destinées à un marché structuré? Ne verrait-on pas, ainsi, prôner l'esprit de «boutiquier" que l'on reproche à juste titre à certains médecins? Il semble que les parlementaires sont de plus en plus nombreux et c'est heureux - à se poser ces questions, exigeant, dans la foulée, une réorientation de la politique suisse en matière de santé. En tant que médecins, nous sommes appelés à y contribuer. Le traitement préconisé pourrait bien aller, pour une fois, dans le sens que nous souhaitons.

\section{Référence}

1 Brunner HH. Révision partielle de la loi fédérale sur l'assurancemaladie; complément à la procédure de consultation. Réponse de la FMH à Madame la Conseillère fédérale Ruth Dreifuss, cheffe du Département fédéral de l'intérieur. Bull Med Suisses 2000;81(35):1887-95. 